\title{
THE RESONANT SCATTERING INTEGRAL; APPLICATION TO THE ANALYSIS OF ELASTIC PROTON SCATTERING
}

\author{
by PHILIP B. SMITH
}

Fysisch Laboratorium der Rijksuniversiteit, Utrecht, Nederland

A relationship is presented which permits a determination of the quantity $\Gamma_{p}^{2} / \Gamma$, where $\Gamma_{p}$ and $\Gamma$ are the particle (proton) and total widths, respectively, of an isolated resonance of which the total spin and channel spin mixing parameter are knowis. The analysis depends upon observation at a zero of the relevant Legendre polynomial, and hence only resonances for which $l \neq 0$ can be analysed. Since the relationship is independent of instrumental resolution, it can be expected to yield better values of the parameters than the usual curve fitting procedure. The results are independent of target thickness, counter efficiency and solid angle, and current integrator precision. The formula given is valid if there is no appreciable $l$-mixing, no overlap of resonances, and if the "hard-sphere" correction to the Rutherford scattering is negligible. Tables are given showing where the latter approximation may be expected to break down. The restriction on $l$-mixing can be removed if the parity allowed $l$ 's are odd, since observation at $90^{\circ}$ (C.M.) makes all interference terms vanish. If the allowed $l$ 's are even it is still possible to apply the formula given if an angle of observation can be found where the interference terms vanish. In both cases difficulty will be encountered in evaluating the results since the $l$-mixing parameter must be known. It is also shown that if the off-resonant scattering can be quantitatively compared to the Rutherford scattering, the range of application of the formula can be extended to cover all well-separated resonances. The extended formula depends, however, on the constancy (not the absolute value) of target thickness, counter efficiency and solid angle, and current integrator calibration. The analysis is applied to published data on the elastic scattering at a bombarding energy of $1.286 \mathrm{MeV}$ in the ${ }^{23} \mathrm{Na}(p, p)$ reaction.

1. Introduction. Elastic scattering of protons has proven to be one of the most fruitful means of investigating resonance levels. The analysis of results is tedious, in general $\left.{ }^{1}\right)^{2}$ ) and is especially so where the level width and the instrumental resolution are comparable. In such cases the purely instrumental parameters are crucial in the analysis and cannot be rigorously known and taken into account without a great deal of work. It is shown that 
the resonant scattering integral provides a simple means of making maximum use of the data to determine the combination of parameters, $\Gamma_{p}^{2} / \Gamma$. This is not all, of course, that may be obtained from elastic proton scattering data. The conventional methods of analysis ${ }^{2}$ ) are not supplanted, but merely supplemented, by this formula. As will become clear in the following exposition, in cases where two channel spins may participate in the formation of the compound state, the mixing parameter must be determined by curve fitting or by another type of experiment. The resonant scattering integral is derived under restrictive conditions in Section 2. The range of validity of the simple formula is treated in Section 3. The conditions under which the restrictions may be removed are discussed in Section 4, and an example of the application of the simple formula is given in Section 5 .

2. The resonant scattering integral. Under the conditions of no $l$-mixing and negligible hard-sphere correction to the Rutherford scattering, the differential cross section for elastic proton scattering in the neighbourhood of an isolated resonance is given by Blatt and Biedenharn ${ }^{3}$ ) as:

$$
\begin{gathered}
\sigma_{p p} \mathrm{~d} \Omega=\left\{\frac{\lambda_{\alpha}{ }^{2}}{8(2 I+1)} \cdot \frac{1}{\left(E-E_{0}\right)^{2}+\left(\frac{1}{2} \Gamma\right)^{2}} \cdot\right. \\
\cdot \Sigma_{j, k} \Sigma_{L}(-)^{s_{j}-s_{k}} \Gamma_{p s_{j}} \Gamma_{p s_{k}} Z\left(l J_{0} l J_{0}, s_{j} L\right) Z\left(l J_{0} l J_{0}, s_{k} L\right) P_{L}(\cos \theta)+ \\
+\frac{\lambda_{\alpha} z\left(2 J_{0}+1\right)}{2(2 I+1)} \cdot \frac{\Gamma_{p}}{\left[\left(E-E_{0}\right)^{2}+\left(\frac{1}{2} \Gamma\right)^{2}\right]^{\frac{1}{2}}} \operatorname{cosec}^{2}\left(\frac{1}{2} \theta\right) \\
\text {. } \left.\sin \left[2 \eta \ln \sin \left(\frac{1}{2} \theta\right)+2 \psi_{l}+\beta\right] P_{l}(\cos \theta)+z^{2} \operatorname{cosec}^{4}\left(\frac{1}{2} \theta\right)\right\} \mathrm{d} \Omega
\end{gathered}
$$

The notation is the same as in reference ${ }^{3}$ ), except that the total particle width is given here by $\Gamma_{p s_{1}}+\Gamma_{p s_{2}}=\Gamma_{p}$, where $s_{1}$ and $s_{2}$ are the two possible channel spins, summed over the indices $j$ and $k$ in the second line of Eq. (1). The second, interference, term (third and fourth lines of Eq. (1)) vanishes at the zeros of $P_{l}(\cos \theta)$. We consider the counting rate vs. energy data at one such angle (preferably the largest) over a fractionally small energy region near the isolated resonance under consideration. The region is assumed to be small enough so that the Rutherford scattering may be considered constant throughout. If we express the instrumental resolution function as $f\left(E-E^{\prime}\right)$, where $E$ is the mean bombarding energy and the function $f$ is assumed normalized to unity, the observed counting rate is:

$$
\begin{gathered}
C(E)=\varepsilon\left\{\frac{\lambda_{\alpha}{ }^{2} \Gamma_{p s_{1}}{ }^{2}}{8(2 I+1)} \int_{0}^{\infty} \frac{f\left(E-E^{\prime}\right) \mathrm{d} E^{\prime}}{\left(E^{\prime}-E_{0}\right)^{2}+\left(\frac{1}{2} \Gamma\right)^{2}} \cdot\right. \\
\cdot \Sigma_{L}\left[Z^{2}\left(l J_{0} l J_{0}, s_{1} L\right)-2 x Z\left(l J_{0} l J_{0}, s_{1} L\right) Z\left(l J_{0} l J_{0}, s_{2} L\right)+\right. \\
\left.\left.+x^{2} Z^{2}\left(l J_{0} l J_{0}, s_{2} L\right)\right] P_{L}(\cos \theta)+z^{2} \operatorname{cosec}^{4}\left(\frac{1}{2} \theta\right)\right\}
\end{gathered}
$$


where the sum over $L$ arises from the second line of Eq. (1) by defining the channel spin mixing parameter $x$ by $\Gamma_{p s_{2}}=x \Gamma_{p s_{1}}$. The cross term is always negative since the two channel spins differ by one unit. The expression used for the instrumental function is legitimate insofar as the absolute width and shape do not change, but merely dislocate, as the energy is changed. Under the conditions of narrow resonances and thin targets this is certainly an excellent approximation. The factor $\varepsilon$ contains the counter efficiency and solid angle, the proton flux, and the target thickness. If $C(E)$ is divided by the counting rate off resonance, $\varepsilon z^{2} \operatorname{cosec}^{4}\left(\frac{1}{2} \theta\right)$, and unity subtracted from the result, one obtains:

$$
S(E)=\frac{\Gamma_{p}^{2} \sin ^{4}\left(\frac{1}{2} \theta\right)}{2 \eta^{2}(2 I+1)} H \int_{0}^{\infty} \frac{f\left(E-E^{\prime}\right) \mathrm{d} E^{\prime}}{\left(E^{\prime}-E_{0}\right)^{2}+\left(\frac{1}{2} \Gamma\right)^{2}},
$$

where $H$ is given by the sum over $L$ in Eq. (2), divided by $(1+\chi)^{2}$.

The curve $S(E)$ is now integrated over all energy, $E$ (in practice only over the narrow range where the integrand differs appreciably from zero). Since the fractional change of $E$ is small, we are justified in taking the slowly varying function of energy, $\eta^{2}$, outside the integral. The result is the resonant scattering integral:

$$
R_{s}=\int_{0}^{\infty} S(E) \mathrm{d} E=\frac{\pi \sin ^{4}\left(\frac{1}{2} \theta\right)}{\eta^{2}(2 I+1)} H \frac{\Gamma_{p^{2}}}{\Gamma} .
$$

This formula makes use of all experimental data in an objective manner, and hence should yield the best obtainable value of $\Gamma_{p}{ }^{2} / \Gamma$ from a given set of data. The result depends on the value of $l, J_{0}$, and $x$. The value of $l$ is assumed known from the outset, since this determines the angle of observation. The values of $J_{0}$ and $x$ are supposed determined by the conventional curve fitting procedure, or from another type of experiment.

3. Range of validity. In writing Eq. (1) three terms of Eq. (7.12) of reference ${ }^{3}$ ) were neglected. These will now be considered so as to set limits to the range of validity of Eq. (4) and also to point to the extension of this formula to quite general conditions. Of the three neglected terms, the easiest to treat is that corresponding to the interference of hard-sphere and resonant scattering. This term contains $P_{l}(\cos \theta)$ as a factor, just as the interference term between resonant and Rutherford scattering (Eq. (1)), and hence is identically zero at the angle of observation. Next we consider the interference term between hard-sphere and Rutherford scattering. This contains the parameters $\phi_{l}$, the hard-sphere phase shifts. In the region of energy in which isolated resonances usually occur, only $\phi_{0}$ is important, and the correction to Eq. (4) resulting from this phase shift is calculated below. The last term to be considered is the pure hard-sphere scattering. 
Since the leading term in this correction is of second order in $\phi_{0}$ it will be entirely negligible as long as the correction above is small.

In consideration of the above, we may write the off-resonant counting rate, or "base line", as:

$$
\begin{gathered}
C(E)=\varepsilon(1-\delta) \operatorname{cosec}^{4}\left(\frac{1}{2} \theta\right), \text { where } \\
\delta=\left(4 \sin ^{2}\left(\frac{1}{2} \theta\right) / \eta\right) \sin \phi_{0} \cos \left(2 \eta \ln \sin \left(\frac{1}{2} \theta\right)+\phi_{0}\right) .
\end{gathered}
$$

This term, $\delta$, has been calculated for several target nuclides over the bombarding energy range in which it changes from a negligible value up to ten to thirty percent. The correction is applied to Eq. (4) by dividing the right-hand side by $(1-\delta)$. The calculations were based on a nuclear radius of $1.15 \times$ $\times 10^{-13} A^{3} \mathrm{~cm}$, which has been shown ${ }^{4}$ ) to give excellent results in the determination of mass differences between mirror nuclei in the mass region 20 to 30 where the present formula is expected to be most useful. Over large regions of energy the correction is seen to be negligible. When it is no longer negligible it must be applied with caution, since it depends strongly on the nuclear radius. The table is given only as a general guide; the correction changes so fast with energy that interpolation is not feasible. Once the correction has reached five or ten percent it is probably a safer procedure to abandon Eq. (4) entirely and use the formula given in the next section.

4. Extensions of Eq. (4). In cases where isolated resonances occur, but the base line differs greatly from the Rutherford value, a formula analogous to Eq. (4) may still be used if the off-resonant counting rate is measured in an absolute manner so that it may be compared to the Rutherford value. Defining this measured ratio as $B$, Eq. (4) is simply modified to:

$$
R_{\delta}{ }^{\prime}=\frac{\pi \sin ^{4}\left(\frac{1}{2} \theta\right)}{B \eta^{2}(2 I+1)} H \frac{\Gamma_{p}^{2}}{\Gamma} .
$$

That this formula is still so simple is due to the fact that the choice of the correct angle of observation eliminates all corrections to the resonant term itself, permitting changes only in the (relatively) energy independent base line. In order to apply Eq. (4') the data should be divided by the base line in the immediate neighbourhood of the resonance, the result diminished by one and integrated, exactly as in the case of Eq. (4).

In practice $B$ can be determined simply if a thin target is used. In this case it is only necessary to determine the factor $\varepsilon$ at low energies where the scattering between resonances is certainly given by the Rutherford value. If the same target is used at high energies, and. if the counter efficiency and solid angle, and current integrator calibration are constant, the factor $B$ is given directly by the ratio of observed to calculated (Rutherford) off-resonant counting rate. There is no necessity of having the instrumental function, 
$t$, constant. On the other hand, additional complications are introduced if the target is thick and the effective target thickness is determined by the

TABLE I

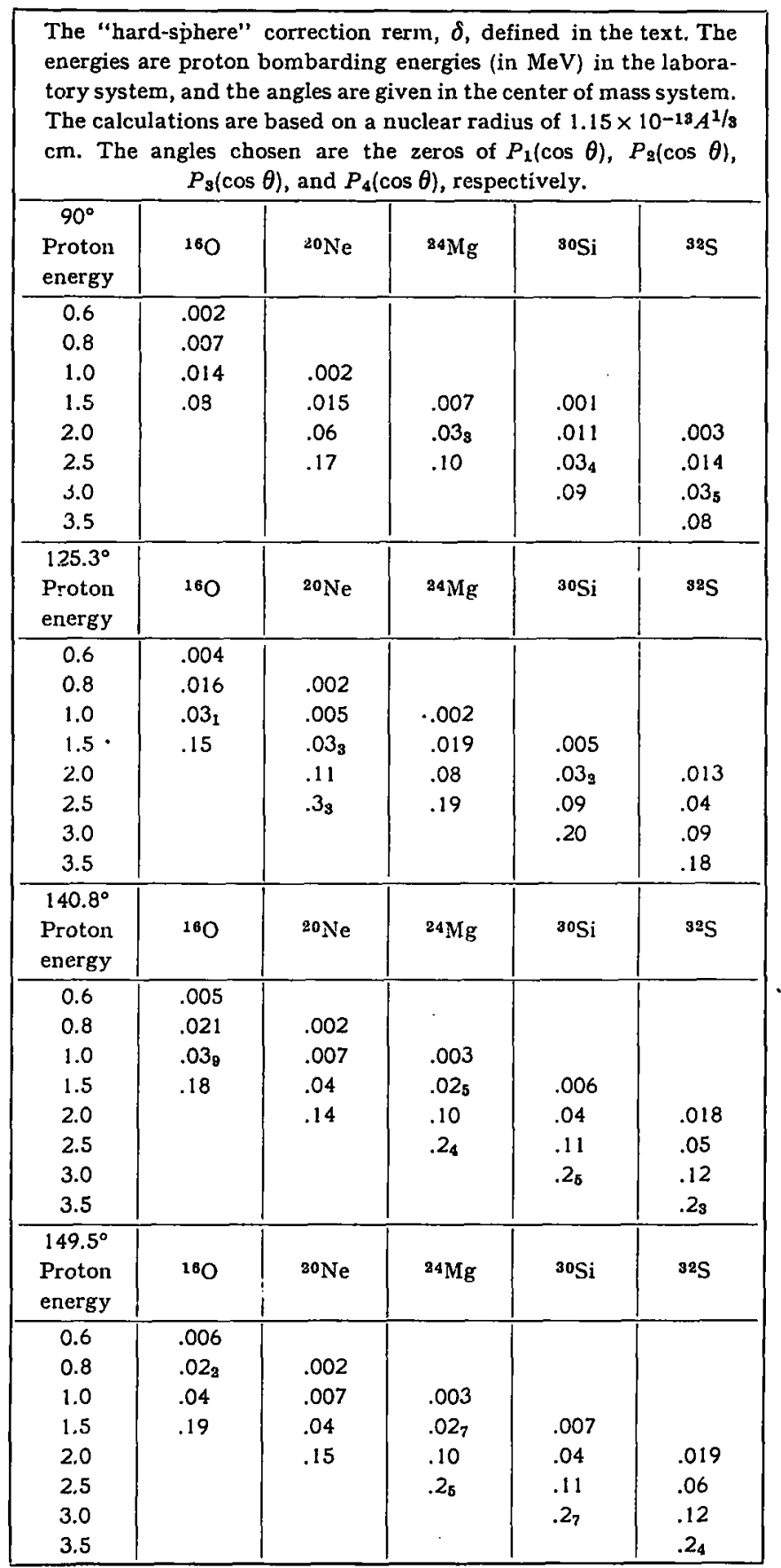


spectrometer resolution and the energy loss in the target. In this case the effective target thickness (which depends on energy loss) is a function of energy.

If $l$-mixing occurs to an appreciable extent the resonant scattering integral can still be applied if an angle is found where the interference terms vanish. Should the parity allowed $l$ 's be odd, observation at $90^{\circ}$ (C.M.) will, of course, lead to the disappearance of all interference terms. If, however, the mixing were to be of $l=3$ with $l=5$, it would be better to seek an angle near the large-angle null of $P_{\mathbf{3}}(\cos \theta)$, since the scattering anomaly will be more pronounced (relative to Rutherford) at larger angles. If there is a mixing of even l's (the only practical case is of $l=2$ with $l=4$ ) it seems likely that an angle can be found near the null of the lower- $l$ Legendre polynomial $\left(P_{2}(\cos \theta)\right.$ in the case mentioned) at which the interference terms vanish. The expression given for the resonant term in Eq. (1) is incorrect if there is $l$-mixing ${ }^{3}$ ). The correct formula is much more complicated and cannot be evaluated unless both of the channel spin mixing parameters, as well as the $l$-mixing parameter are known.

5. An example. The formula of Eq. (4) has been applied to Fig. 7 of the article by Baumann et al.5), giving the elastic proton scattering data at the resonance at $E_{p}=1.286 \mathrm{MeV}$ in the reaction ${ }^{23} \mathrm{Na}+p$. This is a $p$-resonance and the authors state that the observed decays are well fitted by a 1-level assignment. Evaluation of Eq.(4) for this resonance yields:

$$
R_{s}=0.077 H \Gamma_{p}^{2} / \Gamma \mathrm{keV} .
$$

In this case $H$ varies from 2.25 for $x=\infty$ to 2.97 for $x=0$, going through a broad maximum of 3.00 at $x=\frac{1}{4}$. Several values of the channel spin mixing parameter have been reported in the literature $\left.\left.{ }^{5}\right)^{6}\right)^{7}$ ) but fortunately the value of $H$ is remarkably insensitive to this ratio. The data from two runs over the resonance are given in reference 2 , and the numerical integrals (which can be read off, with some care, to $10 \%$ precision) agree within $8 \%$. The final result, using the value $x=2$ given in reference ${ }^{5}$ ) is:

$$
\Gamma_{p}^{2} / \Gamma=5.6 \mathrm{keV}
$$

In Fig. 7 of reference ${ }^{5}$ ) the value of $\Gamma$ is given as $7 \mathrm{keV}$, and the value of $\Gamma_{p}, 4.8 \mathrm{keV}$. These figures lead to $\Gamma_{p}{ }^{2} / \Gamma=3.3 \mathrm{keV}$. These values were obtained from curve fitting. On the other hand, in Table I of the same reference, apparently obtained from different considerations, $\Gamma_{p} \mid \Gamma$ is given as greater than 0.9 , leading to a value of $\Gamma_{p}^{2} / \Gamma$ greater than $5.7 \mathrm{keV}$ and less than $7 \mathrm{keV}$.

A search of the literature revealed no other clearcut case in which the published data and information on the level involved fulfilled the prerequisites for the application of Eq. (4) of Eq. $\left(4^{\prime}\right)$. This appears to indicate 
that in fact the number of resonances which may be so treated is small, but it is felt that this is not the case, since in many cases found in the literature the data had not been obtained at the correct angle, or the level spin and mixing parameter had not been determined.

6. Conclusion. It is felt that the formulas given here will aid in the analysis of elastic scattering data. The form of the integral is familiar in several fields of physics and has proved useful before. It is perhaps worth mentioning that in resonances where only capture and elastic scattering can occur, unique values of the radiation width and the particle width can be obtained from $\Gamma_{p}^{2} / \Gamma$, together with the familiar radiation strength $\Gamma_{p} \Gamma_{\gamma} / \Gamma$.

Acknowledgements. This investigation is part of the research program of the "Stichting voor Fundamenteel Onderzoek der Materie", and was made possible by financial support from the "Nederlandse Organisatie voor Zuiver Wetenschappelijk Onderzoek".

Considerable help in the calculations and in searching the literature has been given the author by J. Kuperus. Many discussions with Prof. Dr. P. M. Endt, who suggested the extension of the formula to the case where $l$-mixing occurs, are gratefully acknowledged.

Received 22-9-'58

\section{REFERENCES}

1) Baranger, E. U., Phys. Rev. 99 (1955) 145.

2) Christy, R. F., Physica 22 (1956) 1009.

3) Blatt, J. M. and Biedenharn, S. C., Rev. mod. Phys. 24 (1952) 258.

4) Van der Leun, C., Thesis, Utrecht, 1958.

5) Baumann, N. P., Prosser, F. W. Jr., Read, W. G. and Krone, R. W., Phys. Rev. 104 (1956) 376.

6) Read, W. G. and Krone, R. W., Phys. Rev. 104 (1956) 1018.

7) Stelson, P. H., Phys. Rev. 96 (1954) 1584. 